\title{
Review of: "De-novo fabrication of sunlight irradiated silver nanoparticles and their efficacy against E. coli and S. epidermidis"
}

Kuo-Pin Yu

Potential competing interests: The author(s) declared that no potential competing interests exist.

This study investigated silver nanoparticles (AgNPs) fabrication by irradiating the raw materials with sunlight using Salvadora persica root extract as a reducing agent. The authors claim this is a "costeffective" and an "eco-friendly" method to fabricate AgNPs in their Abstract. However, the authors did not conduct a cost-effective assessment, and many industrial farmers produce more pollution than we can imagine. This is my first concern. Below are some specific comments

1. Table 1, columns 8: the unit of NPs for ZOI is inconsistent for almost all references and the current study, which makes it very difficult for comparison. Furthermore, the Table Caption should be on the top of the table, isn't it?

2. Figure 2(a): How do the authors confirm the spherical dark images are AgNPs? Did the authors conduct further elemental analysis?

3. Figure 2(d): How do the authors confirm the result of DLS analysis is AgNPs? Did the authors conduct further elemental analysis?

4. The authors claim that the raw material (AgNO3) was bio-reduced to "metallic Ag0" (Discussion, L 3). However, their P-XRD pattern (Figure 3(b)) shows a different result, and the JCPDS file number is incorrect. In the last paragraph, L3 of the Result, Characterization section, the "FCC crystal structure of synthesized SL-SpNPs" should be the "Face-Centered-Cubic (FCC) silver." (The authors should explain what is FCC here, but they did not do it. So, I assured the "FCC" is "Face-Centered-Cubic") The correct JCPDS file number of FCC silver is 04-0783, and the XRD pattern of FCC silver is different from Figure 3(b). 